\title{
Numbers needed to treat derived from meta-analysis: a word of caution.
}

In the editorial by Drs Marx and Bucher in the March/April 2003 issue of Evidence-Based Medicine, ${ }^{1}$ an example illustrating the application of estimates of RRRs to individual risk is misleading. In their example, Marx and Bucher state that the NNT would increase in patients with a baseline risk 11 times that of the low risk group, which is counterintuitive. If a high risk patient is judged to have a risk greater than that of a low risk patient, the NNT would be expected to decrease, not increase. I believe they meant to say that the NNT divided by the factor $\mathrm{f}$ will yield the adjusted risk of outcome in an individual patient.

DANIEL PETERSON, MD

St. Luke's-Roosevelt Hospital Center New York, New York, USA

1 Marx A, Bucher HC. Numbers needed to treat derived from meta-analysis: a word of caution [Editorial]. Evidence-Based Medicine 2003 Mar-Apr;36-7.

\section{Authors' response:}

\section{Dear Dr Peterson:}

Your observation is correct and implicitly concurs with our example, but we failed to correctly state when to divide by factor $\mathrm{f}$ and when to multiply. In paragraph \#6 we wrote: “... the baseline risk of included trials ranged from $2 \%$ to $22 \%$...the corresponding NNT for an average follow-up of 18 months is 263 (CI 185 to 500 ) for a baseline risk of $2 \%$ and 24 (CI 17 to 45 ) for a baseline risk of 22\%."

In paragraph \#7 we wrote: "If the baseline risk of an individual patient is a factor $\mathrm{f}$ compared with the baseline risk of a typical study patient and the relative risk is constant, the ARR for the patient is scaled according to the same factor f." Thus, and as you state, if the baseline risk increases by factor $\mathrm{f}$, the NNT needs to be divided by $\mathrm{f}$. For example, for baseline risk $2 \%$ $\times 11=22 \%$, the NNT becomes $263 \div 11=24$.

Thank you for giving us the opportunity to clarify this point and apologies for any confusion this may have caused.

Marx A, Bucher HC. Numbers needed to treat derived from meta-analysis: a word of caution [Editorial]. Evidence-Based Medicine 2003 Mar-Apr;36-7.

\section{orrection}

the fifth and sixth sentences should appear as follows:

Thus, in our example, if a high risk patient is judged to have ending confidence interval from the original study by the factor $\mathrm{f}$. 